\title{
N-doped graphene quantum dots-functionalized titanium dioxide nanofibers and their highly efficient photocurrent response - ERRATUM
}

\author{
Xiaotian Wang, Dandan Ling, Yueming Wang, Huan Long, Yibai Sun, Yanqiong Shi, Yuchao Chen, \\ Yao Jing, Yueming Sun, and Yunqian Dai
}

doi: 10.1557/jmr.2014.152, Published by Materials Research Society with Cambridge University Press, 30 July 2014.

In Wang et al. ${ }^{1}$, the first corresponding author is listed incorrectly as Yibai Sun. ${ }^{\text {a) }}$ The correct author to whom correspondence should be addressed is Yueming Sun. ${ }^{\text {a) }}$ The email address indicated remains the same: ${ }^{\text {a) }}$ email: Sun@seu.edu.cn.

The publisher regrets the mistake.

\section{REFERENCE}

1. X. Wang, D. Ling, Y. Wang, H. Long, Y. Sun, Y. Shi, Y. Chen, Y. Jing, Y. Sun, and Y. Dai: N-doped graphene quantum dotsfunctionalized titanium dioxide nanofibers and their highly efficient photocurrent response. J. Mater. Res. 29(13), 14081416 (2014). 